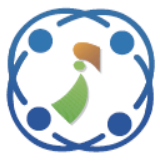

\title{
An Optimal Selection Approach for Mitigating User Time in Cognitive Radio Networks Using Multiple Channels
}

\author{
Sribindu Sattu ${ }^{1 *}$ \\ Moparthy Gurunadha Babu ${ }^{2}$ \\ ${ }^{1}$ Shri Jagdishprasad Jhabarmal Tibrewala University, Jhunjhunu, Churela, Rajasthan, India \\ ${ }^{2}$ Department of Electronics and Communication Engineering, \\ CMR Institute of Technology, Hyderabad, Telangana, India \\ * Corresponding author's Email: sribindu.sattu12@gmail.com
}

\begin{abstract}
In cognitive radio systems; primary clients can use auxiliary clients as agreeable transfers to expand their transmission rates while auxiliary clients will consequently acquire more range to get circumstances, prompting coordinated cognitive radio systems. Earlier research proposals in Coordinated Cognitive Radio Networks (CCRNs) predominantly concentrate on giving omnipresent access and high throughput primary client for clients yet have infrequently contemplated vitality proficiency. Moreover, most existing works expect that auxiliary clients are inactively chosen by primary clients, paying little respect to auxiliary client's eagerness to help, which is clearly not feasible. To address energy issue, this paper identifies an Energy-Efficient Cooperative Strategy (EECS) by leveraging temporal and spatial diversity of the primary network. In particular, auxiliary clients with delay-tolerant packets can proactively settle on the agreeable choices by together thinking about primary channel accessibility, channel state data, primary client's movement stack, and their own particular transmission necessities. Principle challenge for this cooperative relay determination issue is to choose a relay effectively and economically. Since the quantity of auxiliary clients is expanded step by step because of the quickly developing number of wireless communication gadgets, it is unfeasible to examine all the auxiliary clients and after that pick the best. Predominantly primary client transmitter successively watches auxiliary clients. An arrangement of auxiliary clients is chosen in view of transmission criteria. The hand-off choice plan selects the best hand-off from an arrangement of accessible transfers which are chosen in light of transmission criteria of coordinated connections. In the observation of a hand off primary client need to settle on choice in regards to whether to stop its observation and to pick that as best hand-off or to skip to see next transfer. This problem is addressed by optimal stopping theory and optimal stopping rule. To evaluate the performance of proposed scheme Optimal Selection Approach (OSA), it is compared with auxiliary client's energy efficient and existing cooperative schemes. Extensive simulation study is conducted so that impacts of different system parameters are investigated and algorithm proposed can satisfy different system requirements.
\end{abstract}

Keywords: Multiple channels, Energy efficiency, Cognitive radio networks (CRNs), Cooperative communication, Relay selection, Optimal observation order, Optimal selection approach.

\section{Introduction}

Cognitive radio (CR) is an important technique to improve utilization efficiency of scarce spectrum [1]. According to a recent survey [2], the current Information and Communication Technology (ICT) industry is responsible for about $2-4 \%$ of the entire carbon footprint generated by human activities, which corresponds to about $25 \%$ of all car emissions and is approximately equal to that for all airplanes. In light of this, the trend of reducing energy consumption motivates researchers to explore novel technologies to achieve energy-efficient "Green" communications. One of the feasible solutions is to utilize the spectrum more efficiently. The reason lies in the Shannon's capacity, which reveals the tradeoff between power and bandwidth. Specifically, link capacity increases only logarithmically with 
power but linearly with bandwidth, which indicates that more bandwidth brings down power consumption. In [3], Holland also showed that more than $50 \%$ of power can be saved by dynamic spectrum management. Hence, the cognitive radio (CR) technology, which can be traced back to Mitola in 1999 [4], can be applied to improve the spectrum efficiency and thus reduce the energy consumption.

In a CR network, users are rational and capable of sensing the dynamic environment, and adaptively adjusting their transmission parameters based on the interactions with other surrounding users. [5]. The CR technology can be applied to address the unbalanced usage of spectrum by allowing unlicensed secondary users (SUs) to opportunistically access the spectrum bands unoccupied by licensed primary users (primary clients). Generally, CR devices are designed based on software-defined radios to allow SUs to have the capabilities to sense, learn and adapt to wireless environments and then coexist with primary clients in such a way that SUs' transmissions will not interfere with Primary clients [6]. There are three paradigms to make the coexistence of primary clients and auxiliary clients possible: underlay, overlay and interweave [7]. The underlay paradigm allows SUs to transmit simultaneously with primary clients as long as the interference to primary clients is below a certain threshold. In the overlay paradigm, auxiliary clients obtain spectrum from primary clients by helping maintain or improve the communication of primary clients with sophisticated signal processing and coding. In the interweave paradigm, auxiliary clients opportunistically access spectrum holes without interfering primary clients. With the evolution of all these paradigms, the spectrum efficiency can be improved significantly, and hence reduces auxiliary clients' energy consumption.

In the case of low SNR, relying on one auxiliary client to detect the presence of primary client is highly unreliable. To improve the sensitivity of CR spectrum sensing even further, and to make it more robust against fading and the hidden terminal problem, cooperative sensing can be used. The concept of cooperative sensing is to use multiple sensors and combine their measurements into one common decision. This approach including soft combing and hard combing that are used most widely [20, 21].

Here the problem of cooperative relay selection in a CRN is focused where a primary client pair observes the SU candidate relay nodes based on an observation sequence and decides whether to stop and select the current auxiliary client node under observation as the cooperative relay node. The primary client pair makes the decision based on comparing the result of the instantaneous reward and the expected reward of future observations to maximize the reward of the selection. The instantaneous reward can be indicated by the channel quality of the candidate relay being observed, and the reward without bounds observations is the reward that the set of primary client can acquire if it maintains monitoring the below candidate transfers. In other words, the primary client pair may stop and receive the current reward/relay or continue observing the rest of the auxiliary client relays to find a better relay. Since an auxiliary client can only obtain current, not the future, information of primary network, it needs to decide at which time slot to stop observing and with which primary client to cooperate in order to maximize its energy efficiency. In this section, we first propose auxiliary client's spatial cooperative strategy is defined as an instantaneous reward (i.e., energy-efficiency) at current time slot. At last, the issue of coordinated relay choosing is described as an ideal layoff issue.

Definition 1: An optimal stopping problem is defined by two sections:

- A set of arbitrary variables, i.e., $X_{1}, X_{2} \ldots \ldots$ with a known joint distribution; and

- A sequence of reward functions, i.e., $Y_{1}\left(X_{1}\right), \ldots \ldots . Y_{\alpha}\left(X_{1}, X_{2}, \ldots \ldots\right)$, which are the real-valued functions of the variables previously discussed.

The objective is to find out the variable $X_{\mathrm{i}}$ in the sequence such that the reward function indicated by $Y_{i}\left(X_{1}, X_{2}, \ldots \ldots\right)$, is maximized and is described as in Eq. (1).

$$
0<(1-\alpha) R_{p s}^{r}(t) \leq \alpha \beta R_{s p}^{r}(t)
$$

Where $R_{p s}^{r}(t)$ represents the transmission rate among the primary client transmitter and the SU relay, and $\mathrm{R}_{\mathrm{sp}}^{\mathrm{r}}$ represents the transmission rate among the SU relay and the primary client receiver. $\alpha$ - Constant value of secondary relay node, $\beta$ secondary node relay's data. The intuition behind this equation is that the amount of data transmitted from the primary transmitter should not exceed the transmitting capability of the relay.

The value of $\alpha$ is controlled by the primary client transmitter. Given $\alpha$, minimum value of $\beta$ can be derived as $\beta_{\text {low }}=(1-\alpha) / \alpha$ when it is assumed that $R_{p s}^{r}(t)=R_{s p}^{r}(t)$. Here $0.5 \leq \alpha \leq 1$ and $0 \leq$ $\beta_{\text {low }} \leq 1$. 
In a cognitive communication network, an SU may not be available to serve the primary clients due to the secondary communication carried out by auxiliary clients. Therefore, the primary client transmitter should examine the availability of the auxiliary client candidate relays. When the primary client transmitter observes the channel condition of an auxiliary client, the auxiliary client returns a value for $\beta$. It is assumed that the auxiliary client is always in saturated transmission mode, which means that the auxiliary client candidate relay never returns a parameter $\beta=1$.

In CRNs, cooperative communications can be classified into two categories:

i. Cooperation among auxiliary clients;

ii. Cooperation between primary clients and auxiliary clients.

In this paper, we will focus on the latter where SUs firstly serve as cooperative relays to help primary clients finish their transmissions earlier, and in return obtain the primary clients' channels as a reward [8, 9]. This system is well-known as cooperative cognitive radio networks, wherein the mutual advantage among primary clients and auxiliary clients is realized.

The cooperation communications between primary clients and auxiliary clients in CCRNs have been widely studied in the literature [8 - 17], most of which focused on hand over ubiquitous approach and huge throughput for both primary clients and auxiliary clients. Besides, some of them have an implicit premise that SUs will be engaged in cooperation as long as some forms of rewards are guaranteed by primary clients, such as throughput, energy-efficiency, or in general utilities (e.g., a function of reward and payment). However, in practice, without incentives, auxiliary clients may not be interested in cooperation if they have lightloaded and delay-tolerant traffic or if the received rewards from $\mathrm{CC}$ are no more than what will be obtained by directly using primary clients' idle channels in the future. Therefore, SUs may hold their transmissions for later time instead of cooperating immediately.

In addition, to further stimulate "Green" communications in the context of CCRNs, there requires more efforts on studying the energy efficiency of auxiliary clients other than that of primary clients. The reason is that the number of secondary wireless devices is and will be significant in current and foreseeable ICT industry and these devices only have limited battery storage while the energy dissipation rate is one of the primary concerns for users. However, among recent research work in CCRNs, developing an energy-efficient cooperative strategy from SUs' perspective has rarely been investigated carefully as yet. Therefore, in this paper, we attempt to address this issue by exploring auxiliary clients' cooperative strategy in CCRNs with the objective to maximize their energy efficiency. Specifically, we consider a time-slotted CCRN where auxiliary clients have delay-tolerant packets to transmit by a certain deadline. auxiliary clients observe the primary network slot by slot in time sequence and make the cooperation decision in both temporal (i.e., what time) and spatial (i.e., which primary client) domains. In traditional (noncognitive radio) multiple-relay networks, three relay protocols (i.e., fixed relaying, selection relaying and incremental relaying) have been studied extensively in [10]. In particular, this paper firstly discusses optimal cooperative strategy when SUs attempt to transmit fixed amount of packets, and then explore the one when auxiliary clients have continuously new arriving packets during the decision process. Our major contributions are summarized as follows:

- This study focuses on auxiliary clients' energyefficient cooperation strategy in CCRNs with consideration of primary clients' temporal and spatial diversity in channel availability and traffic load.

- In this paper, we consider of first one is that SUs schedule the transmission for fixed amount of packets. The other one is that SUs have continuously new arriving packets during the observation and decision process and the transmission deadline is dynamic depending on auxiliary clients' buffer overflow probability. The decision related problems for both scenarios are formulated based on the optimal stopping theory and the backward induction is applied to derive their corresponding optimal strategies.

- We thoroughly investigate auxiliary clients' optimal cooperative strategies and concentrate on signaling overhead and delay. The constraint of control signal exchange for resource optimization is another factor to be observed. It is observed that, controlling signal overhead is high is spectrum sensing and allocation under heterogeneous network condition. This control signal exchange introduces delay in the network, degrading the performances.

- We consider the main attributes, it as Access time, Spectrum availability and Relay node time. In the present CRN approach, the spectrum allocation is limited to the registered network region, and the entire spectrum available out of a network bond region remains utilized. 
The paper is organized as follows. Section 2 presents related work. The proposed framework is mentioned in section 3. Section 4 describes the experimental study of proposed paper. And finally the paper is concluded in section 5 .

\section{Related work}

In recent years, researchers have devoted great efforts to developing CCRNs from different perspectives. Simeone propose a cooperation scheme wherein primary clients' transmission rates are maximized with help of SUs and certain time slots are in return leased to SUs, which afterwards compete for transmissions following a distributed power control mechanism [11]. Zhang improve the work in a way that primary clients can collect revenues from SUs when primary clients' transmission rates are satisfied and TDMA is adopted as SUs' access model for primary channels [12]. Cao in [13] study the optimal communication strategy for primary clients by quantifying the impacts of energy and power consumptions on primary clients' utilities. These works formulate hierarchical Stackelberg games where primary clients are the leaders who make the cooperation rules while auxiliary clients as a group of followers obey the rules. Besides, some other work focuses on searching for secondary relays for primary clients. $L i$. in [14] considers a multi-hop secondary relay selection problem, in which the path is complicated via performing primary clients' strategies. In [15], Jing model the relay selection problem using optimal stopping theory to guide primary clients to find the SU relay that can maximize the throughput for primary clients. However, in aforementioned papers, SUs are considered not to proactively choose their cooperative strategies, which may not be appropriate considering auxiliary clients are rational or selfish.

To address this issue, there exists several works studying cooperative schemes from auxiliary clients' perspective. In [16], Jayaweera propose both centralized and decentralized decision making architectures for SUs to place bids indicating how much power they are willing to spend for relaying primary clients' traffic. Zou in [17] study a new CCRN where primary clients lease their spectrum as well as transmit power to relay data for SUs while primary clients in return collect revenues from auxiliary clients. The spectrum access and power allocation problem is formulated as an auction model to maximize SUs' aggregated throughput. In [9], Long focus on secondary network throughput maximization by allowing auxiliary clients to optimally share the cooperation generated spectrum resources. In [18], Zhang exploit a novel CCRN paradigm with antenna polarization and energy harvesting capability, which allows auxiliary clients' own data. Cao in [19] apply this CCRN model and achieve maximization of the weighted sum throughput of primary clients and auxiliary clients using dynamic Markov decision process subject to energy constraints.

In [24], an objective work is assessed for each stream by expanding the need with channel pick up. Keeping in mind the end goal to store the target work an incentive in diving request and afterward appropriated to the separate kind of SU (secondary user). The stream with most elevated target work is assigned to the MRDG, trailed by MRG (minimum rate guarantee) and MDG (minimum delay guarantee). The stream with slightest target work is appointed to BE (best effort service).

In [25], application of the heterogeneous measurable QoS driven power designation plan to amplify the successful limit of the MIMO-OFDMA based handing-off model with heterogeneous factual deferral bound QoS prerequisites.

Although auxiliary clients' energy-efficiency issues have been investigated in some of these literatures, our scheme is distinct from them in that SUs exploit the temporal and spatial diversity of the primary network and proactively make cooperative decisions based on their own transmission requirements (e.g., transmission deadline, buffer overflow probability, and etc.). Furthermore, we capture the feature of time non-reversibility in decision-making process and apply optimal stopping theory to drive auxiliary clients' cooperative strategy. In particular, we explore the impacts of two practical scenarios on SUs' cooperative strategy and demonstrate that the achieved energy-efficiency outperforms what is obtained using conventional cooperative schemes.

\section{Proposed framework}

In this paper, we proposed new solution for cognitive radio networks. It is very effective and energy efficient. We incorporate this mechanism named as optimal selection-oriented mechanism. This is derived as the solution to cooperative relay selection problem. To select a user before knowing position of user and knows efficiency of user. In our work, we are used two users. Second user transmission depends on first user transaction process. We can know the network process, check whether energy levels of users sufficient for next process or not. The solution of our approach is 
formulated as backend process in network. The maximum return that the primary client transmitter can obtain after obtaining $j_{t h}$ candidate relay is denoted by $V_{j}^{(M)}\left(x_{1}, x_{2}, \ldots x_{j}\right)$, and it is described as in Eq. (2).

$$
\begin{aligned}
& V_{j}^{(M)}\left(\mathrm{x}_{1}, \mathrm{x}_{2}, \ldots \mathrm{x}_{\mathrm{j}}\right) \\
& =\max \left\{y_{j}\left(x_{1}, x_{2}, \ldots x_{j}\right)\right\} \\
& E\left\{\mathrm{~V}_{\mathrm{j}+1}^{(\mathrm{M})}\left(\mathrm{x}_{1}, \mathrm{x}_{2}, \ldots \mathrm{x}_{\mathrm{j}+1}\right) \times \mid \mathrm{x}_{1}=\mathrm{x}_{1}, \mathrm{X}_{2}=\mathrm{x}_{2}, \ldots, \mathrm{X}_{\mathrm{j}}=\mathrm{x}_{\mathrm{j}}\right\}
\end{aligned}
$$

Where $y_{j}\left(x_{1}, x_{2}, \ldots x_{j}\right)$ represent the instantaneous reward after $\mathrm{K}_{\text {th }}$ observation, and it is described as in Eq. (2)

$$
E\left\{V_{j+1}^{(M)} \mid X_{1}=x_{1}, X_{2}=x_{2}, \ldots, X_{j}=x_{j}\right\}
$$

Eq. (3) represents the expected reward achieved by skipping to observe next $\mathrm{SU}$ relay.

When $V_{j}^{(M)}\left(x_{1}, x_{2}, \ldots x_{j}\right)=y_{j}\left(x_{1}, x_{2}, \ldots x_{j}\right)$

It is optimal to stop scanning the relays. The optimal selection-oriented rule is achieved when the following condition is satisfied

$$
\begin{gathered}
y_{j}\left(x_{1}, x_{2}, \ldots x_{j}\right)>E\left\{V_{j+1}^{(M)}\left(x_{1}, x_{2}, \ldots x_{j+1}\right) \times\right. \\
\left.\mid X_{1}=x_{1}, X_{2}=x_{2}, \ldots, X_{j}=x_{j}\right\}
\end{gathered}
$$

Backend process is clearly understood when the expected reward, $E\left\{V_{j+1}^{(M)}\right\}$

Is defined as $Z_{M-j}$, if primary client pair proceeds to scan next SU relay which is given and described as in Eq. (4)

$$
\begin{aligned}
& Z_{M-j}= \\
& \quad E\left\{V_{j+1}^{(M)}\left(x_{1}, \ldots x_{j+1}\right) \times \mid X_{1}=x_{1}, \ldots, X_{j}=x_{j}\right\}
\end{aligned}
$$

The set $\left\{X_{l}, X_{2 \ldots} X_{M}\right\}$ for SU candidate relays are mutually independent. Thus $Z_{M-j}$ is a constant that only depend on $\mathrm{M}-\mathrm{j}$, the number of remaining steps to continue. Optimal stopping rule as follows. Primary client pair observes candidate relays based on observation sequence $\mathrm{S}$ and obtain instantaneous reward $y_{k}$ after $k^{\text {th }}$ observation. Then the value of $y_{k}$ with the value of $Z_{M-k}$ is compared and decide to stop at $k^{\text {th }}$ step if $y_{k}>Z_{M-k}$ and to continue observing next relay otherwise. If the primary client pair observe the last relay in the observation

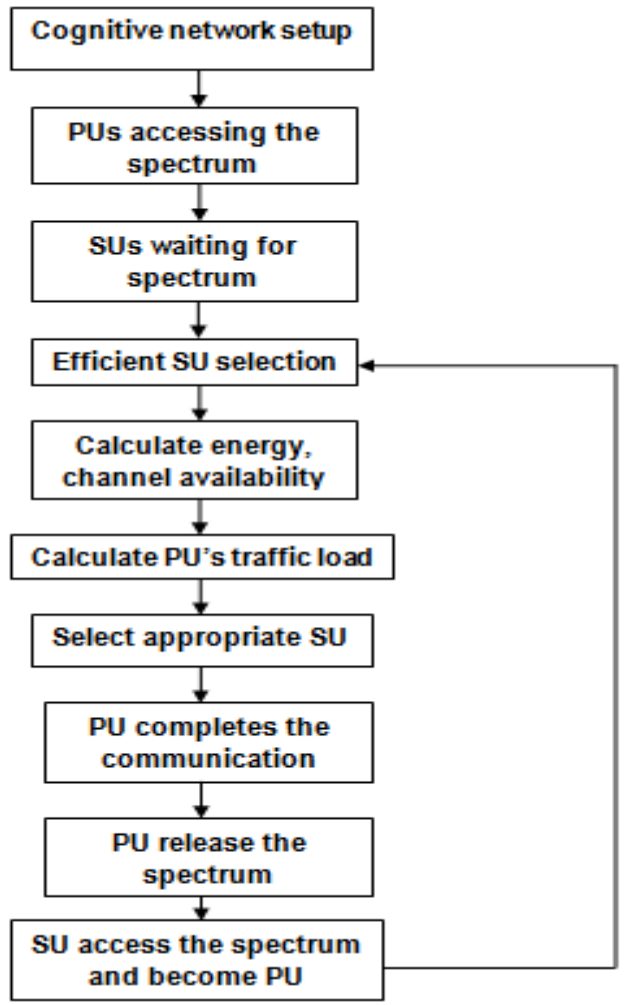

Figure. 1 Proposed framework

sequence and the condition is not satisfied and it is forced to take that last relay in the sequence as cooperative relay. This is called wrong relay selection.

If the primary client pair stops and selects a suitable cooperative relay more quickly when observing the SU candidate relays based on an observation order denoted by $\mathrm{C} 1$ compared with another observation order denoted by $\mathrm{C} 2$, say that the order $\mathrm{C} 1$ is more efficient than the order $\mathrm{C} 2$. First, consider a random observation order strategy in which the primary client pair constructs the observation sequence randomly at every time slot. Since the observation variable set $\left\{X_{1}, X_{2} \ldots X_{M}\right\}$ is independent for each time slot, the efficiency of this order strategy is uncontrollable.

In our cognitive radio network, all users deployed and configured for specific reason behind the system process shows in Fig. 1. Initially, primary users are accessing the spectrum, so this particular time secondary users waiting for spectrum. Here we proposed a new solution for efficient the secondary users selection. In this system, we consider some parameters like energy, channel availability and primary client's (primary users) traffic load and do the metric wise calculation. Here based on parameters, select the appropriate secondary user then configure the setup of network. Whenever complete the primary client 
communication, in particular time primary client releases the spectrum. In our solution, secondary user (SU) accesses the spectrum and become primary user (primary client). The secondary users can efficiently connect with their destination nodes with less waiting time from primary users. In our proposed framework, the reduced easy waiting time improves the overall network efficiency and also the energy efficient relay node selection improves the spectrum usage and availability.

\section{Experimental study}

Experimental setups: Our experiments are conducted using the NS-2 simulator. We conduct the experiments in two steps. The initial step is to check the viability of our plan, and then deeper study is investigation is done to assess the cost and overhead in more detail. In the first step, there are 10 mobile nodes in the network, and four nodes are considered as sources and five nodes are considered as destinations. The individual traffic is concurrently to the same destination node. The channels used for radio network is five. We consider length of packets in individual node is 50 .

All of the nodes randomly move at an average speed of $10 \mathrm{~m} / \mathrm{s}$. The simulation time is $150 \mathrm{~s}$; check the primary user's information and availability of spectrum. Here we set area for random way communication is 500x500. The connections among mobile nodes are TCP connections, and we send FTP (File Transfer Protocol) traffic in each communication channel. The FTP rate of the connections is $512 \mathrm{~Kb} / \mathrm{s}$, and the threshold of the user is $1 \mathrm{M} / \mathrm{s}$, so two primary users sending the traffic to the same destination user will not cause an alert but secondary users will. The size of the scenario field is $1000 \mathrm{~m} \times 1000 \mathrm{~m}$. The queue drop mechanism is tail drop. The routing protocol we use is a revised Ad-Hoc On-Demand Distance Vector (AODV) routing protocol that integrates our optimal selection method.

Table 1. Network scale configurations

\begin{tabular}{|c|l|c|c|}
\hline $\begin{array}{l}\text { Node } \\
\text { number }\end{array}$ & $\begin{array}{l}\text { Field size } \\
(\mathrm{mxm})\end{array}$ & $\begin{array}{l}\text { Number of } \\
\text { primary and } \\
\text { secondary } \\
\text { users }\end{array}$ & $\begin{array}{l}\text { Number } \\
\text { of } \\
\text { channels }\end{array}$ \\
\hline 2 & $100 \times 100$ & 1,1 & 1 \\
\hline 4 & $250 \times 250$ & 2,2 & 2 \\
\hline 8 & $500 \times 500$ & 2,4 & 5 \\
\hline 16 & $1000 \times 100$ & 6,8 & 8 \\
\hline 32 & $1500 \times 1500$ & 12,14 & 14 \\
\hline
\end{tabular}

In step two, we assess the execution of our new optimal selection scheme in a different network scale. The configurations of different network scale are shown in Table 1. The traffic of node is applicable to our network process.

Three metrics are adopted to conduct a comparison study between our optimal selection of user approach, optimal stopping rule and the auxiliary client's energy efficiency mechanism. The main purpose is to check how much signal overhead has been caused in order to complete the primary client communication process. The three metrics are defined as below:

- Packet propagation delay: average time for one packet to propagate from the source node to destination node.

- Energy levels of node: the energy level of nodes uses per second.

- Network performance: the number of transmitted packets calculated in Mega bits per sec

Fig. 2 displays deployment of users in cognitive radio network and all users to verify the spectrum availability. Here the total users communicate through channels.

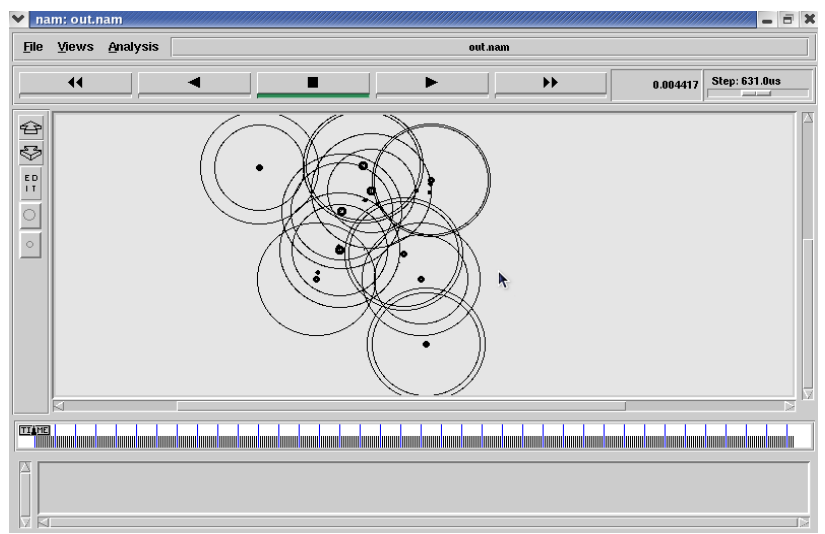

Figure. 2 The cognitive network topology

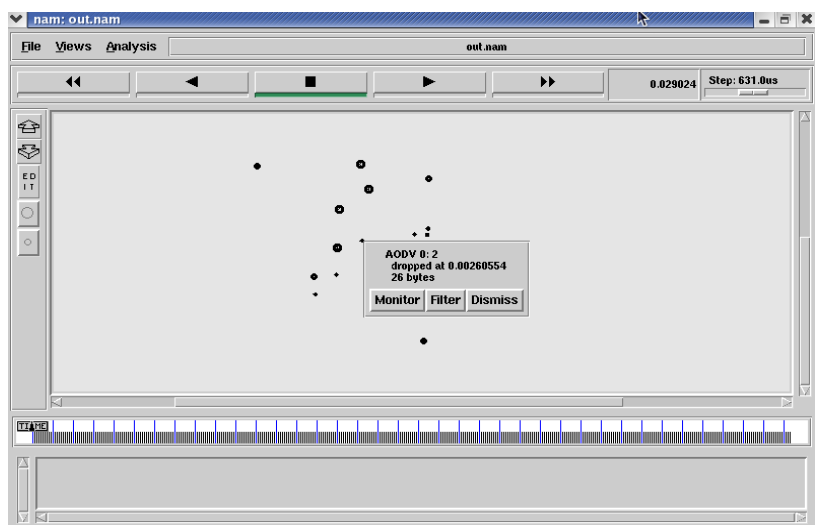

Figure. 3 The system checking the availability of spectrum 


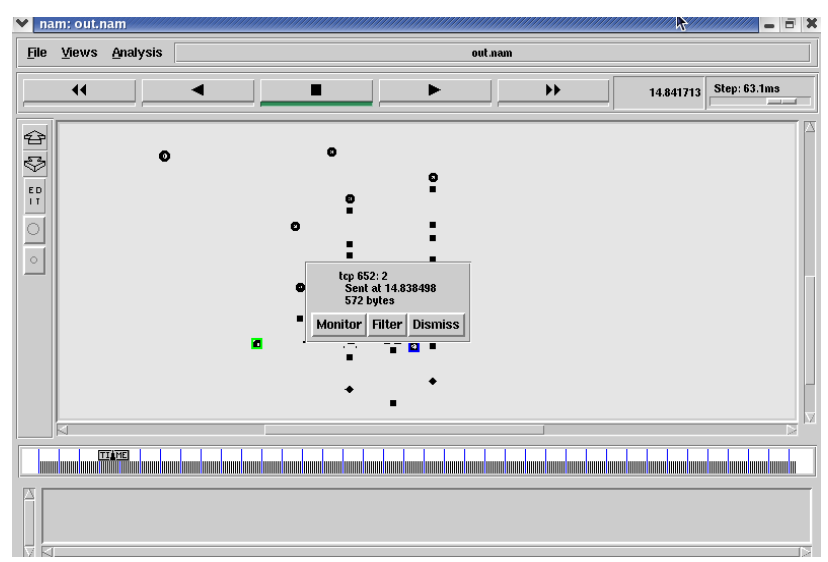

Figure. 4 The data process between users

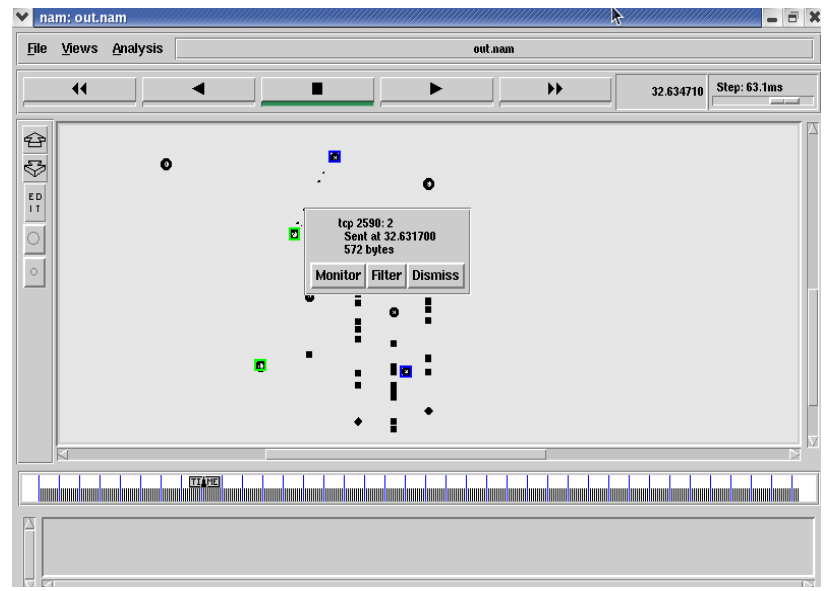

Figure. 5 Primary user sending the data by accessing spectrum

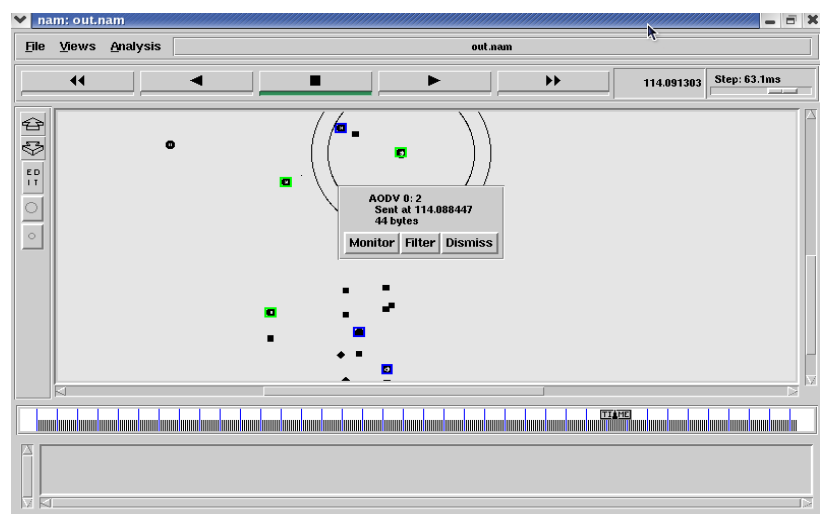

Figure. 6 Data process between users based on routing

Here the availability of spectrum is verified and routing process is depends on protocol as working radio network. The spectrum availability checking probability indicates through dropping rate of data process as shown in Fig. 3.

The data process between users is seen in Fig. 4. The second users are in waiting condition until primary users completes the transmission of data. In this NAM output, primary user accesses the spectrum and starts the sending of data as represented and data is processed through TCP
(Transmission Control Protocol) protocol and it is accessible at system level. The bytes of data are seen in Fig. 5.

In the data level process, the protocol verifies the routing process whether the spectrum is available or not and further looks for the next communicating level for secondary users. Fig. 6 shows the routing level data process.

Fig. 7 displays link capability, bandwidth and delay. Here the routing delay is depends on user waiting time for spectrum. From the above figure the link between the nodes 1 and 9 is observed, and the bandwidth is measured as $10 \mathrm{Mbits} / \mathrm{sec}$. The delay is observed as $10 \mathrm{~ms}$ based on routing level.

The data process numerical results are shown in Fig. 8. As per the results, total number of data packets sent is 18949 , in which 14284 are delivered. We observed the zero packets dropping in spectrum, but the overall dropping rate is 0.00754 .

Deployment of the total users in the network based upon the topology values are shown in Fig. 9.

Fig. 10 displays the broadcasting process of network. It is clearly observed that, each node is communicating to transfer the data in between each other. The broadcasting process is useful to find the level of routing and to find the destination of data to where it is transferred from nodes.

In this proposed system, we used to check the availability of spectrum, considering of parameters for creation of channel and to find routing process.

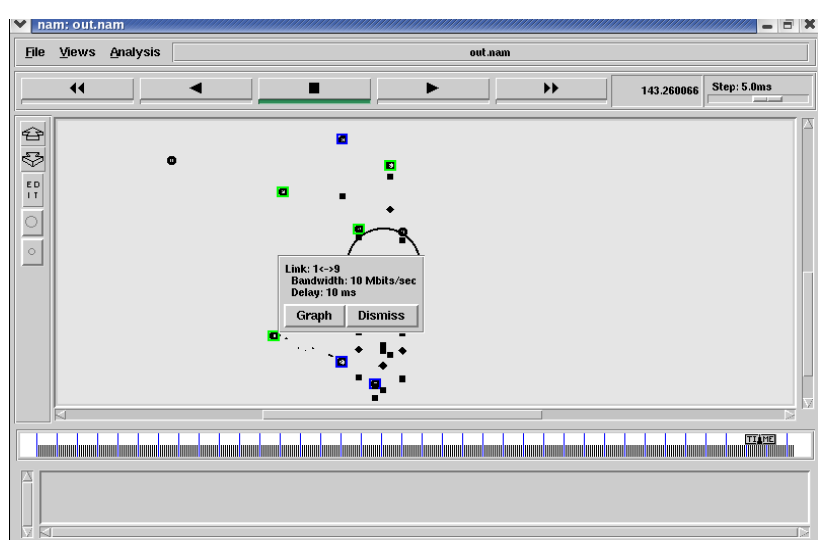

Figure. 7 Network data process (links, bandwidth, and delay)

simulation_result.csv $\times$

Messages Sent, 18949

Messages Recieved,14284

Messages Dropped, 0

Drop Rate,0.00753812866114307

Drop Rate,1.14272

Figure. 8 Numerical results of data process 


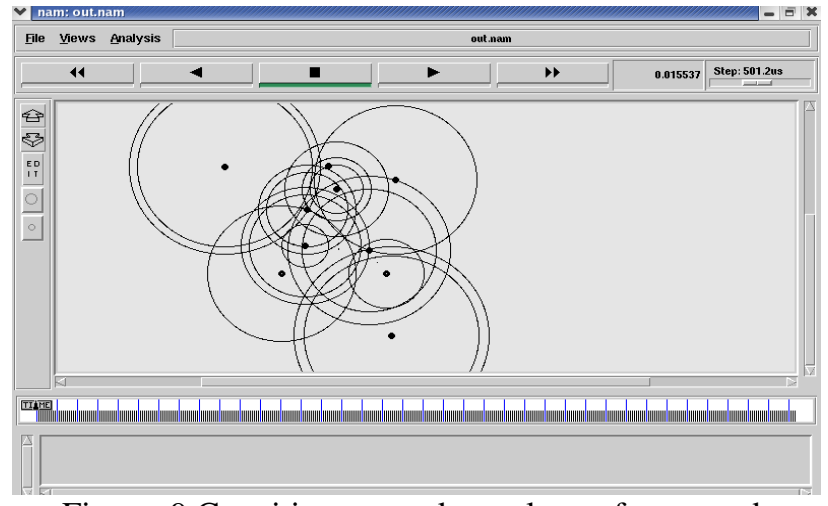

Figure. 9 Cognitive network topology of proposed scheme

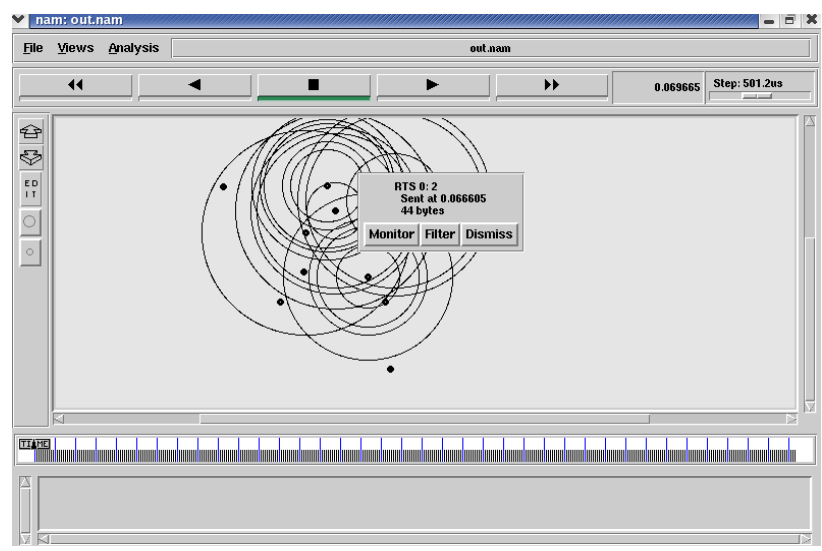

Figure. 10 Broadcasting of network process

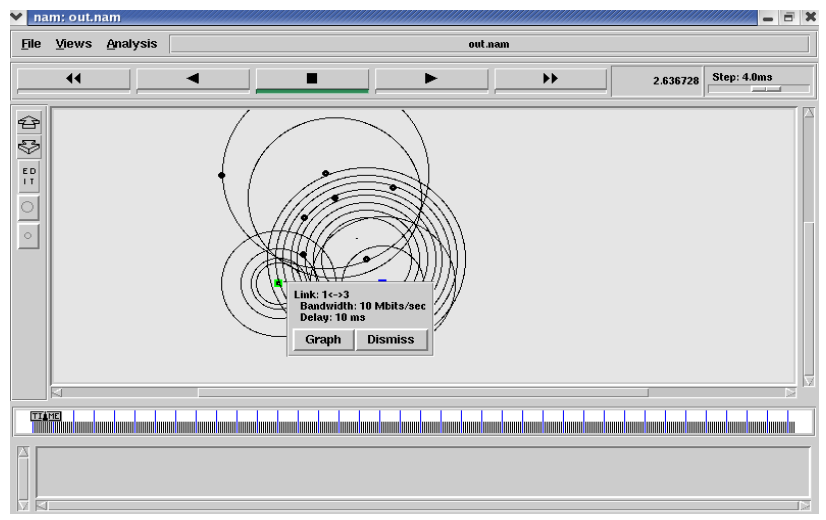

Figure. 11 System verifying availability spectrum

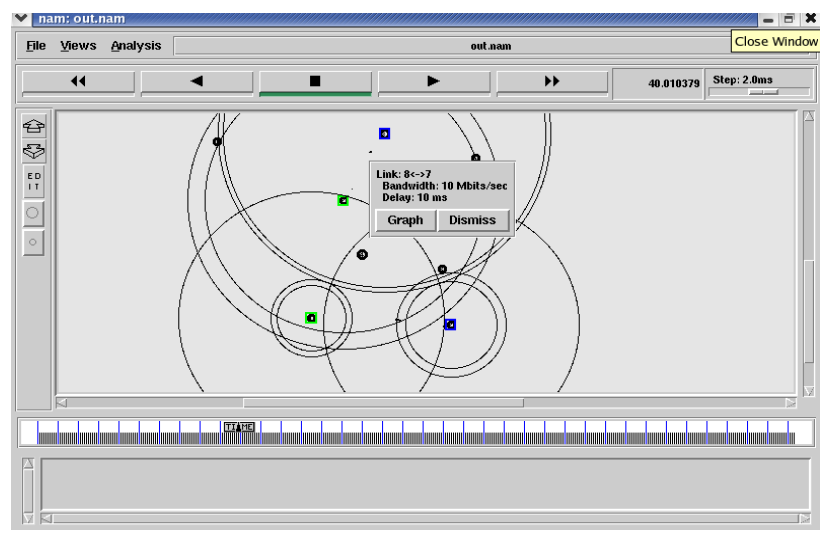

Figure. 12 Secondary user sending data without wait time

\section{simulation_result.csv $\times$ \\ Messages Sent,27005 \\ Messages Recieved,26849 \\ Messages Dropped, 0 \\ Drop Rate,0.00994223291982966 \\ Drop Rate,2.14792}

Figure. 13 Numerical results of proposed data process

It is observed that, the proposed system is having an advantage of secondary user waiting time is reduced based on primary user transmission process completion. Here, the routing process depends upon protocol and it is shown in Fig. 12.

The simulation results of proposed system are mentioned in Fig. 13. As per the results, total number of data packets sent is 27005, in which 26849 are delivered. We observed the zero packets dropping in spectrum, but the overall dropping rate is 0.00994 .

Result analysis: The first experiment is used to verify the effectiveness of our strategy from the energy efficient cognitive radio network. And also the second step is managed to determine the energy levels, particularly the performance of network process compared to the optimal stopping rule and the auxiliary client's energy efficiency mechanism.

Verification of effectiveness: In the first experiment, 10 nodes are randomly generated, and one of the topologies is shown in Fig. 2. In our simulation process, we considered primary users and secondary users check the availability of spectrum based upon routing process.

During the simulation, we considered random topology and different channels taken for communicating between users. Here, we check the spectrum availability first and knowing the status of user availability. The data process can be starts from primary user and secondary user wait until complete the process of primary user. Therefore, during the simulation, we have observed that the secondary users have tried to check the spectrum availability, but the primary user doesn't complete the data process within time.

The simulations results have verified that our proposed system is capable of overcome the transmission time of primary user to complete the data process and allowing the secondary user routing process. In addition, it has also shown that the protocol can recover the waiting time of secondary users.

Performance analysis: The experimental analysis given and unveils that the correlation with the Energy efficient cooperative strategy (EECS) with the agreeable procedures calculations, for 
example, the calculation given in [23] like channel state information, helpful correspondence, and choice of optional client. According to the author's worry the framework system contrasted with cooperative strategy (CS) calculation given in [22] like optional clients, utilizing hand-off choice, channel pick up and security. Here our paper introduces and examined proposed structure contrasted with existing development calculation is better.

Figs. 14 to 16 represents the experimental results of the three performance metrics measured in our new proposed scheme, the optimal stopping rule and the SU's energy efficiency mechanism. The lines with green color present the performance of new routing mechanism, while the lines with red color present the performance of modified AODV as the optimal stopping rule and blue color present the performance of SU's energy efficiency mechanism.

The experimental results have shown that the impact of SU's packet arrival rate on its spectral efficiency in different primary network settings. Meanwhile, Fig. 14 shows that the delay increased because of waiting time of secondary user is increasing. When a normal user wants to communicate with a primary user, it will buffer some packets first and then start the route discovery process. As one primary user only has one spectrum available at certain time, the broadcasted (Route REQuest) RREQ may spend more time finding it and will include it in the route. For instance, in our experiment, the RREQ traversed around almost half of the network to find the spectrum availability. As a result, the first buffered packets will be delayed for a longer time before getting to the receiver. With the increase of the number of primary users and network scale, the probability of this situation increases and its effects on average delay are more obvious.

Our protocol does not affect the less number packets delivered per minimum time interval. Fig. 15 shows that, with the increase of packet arrival rate, auxiliary client's throughput are improved. Moreover, for a certain packet arrival rate, auxiliary client's spectral efficiency increases when the primary network incorporates more primary clients. Note that the previous scenario equals to the case where the packet arrival rate is zero. It can be observed that a higher spectral efficiency is achieved in the scenario of auxiliary client with continuously arriving packets than that in the scenario of auxiliary client without newly arriving packets, as shown in Fig. 15. Fig. 16 shows that, individual node energy levels based on network routing process and routing levels more efficient it will be effect to process of routing levels.

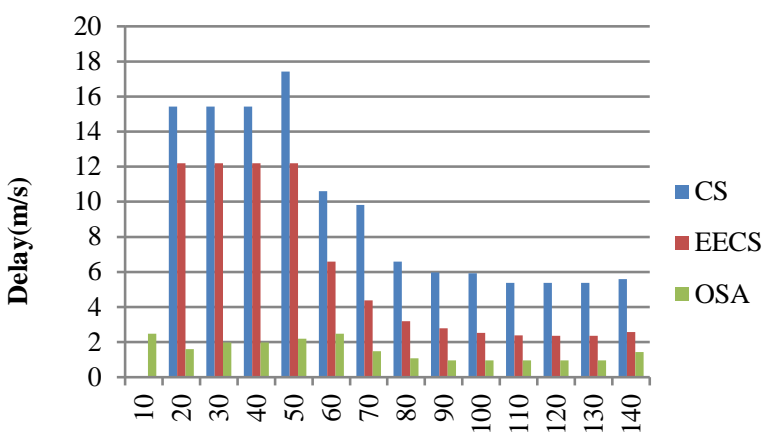

Time (s)

Fig. 14. Delay time in network

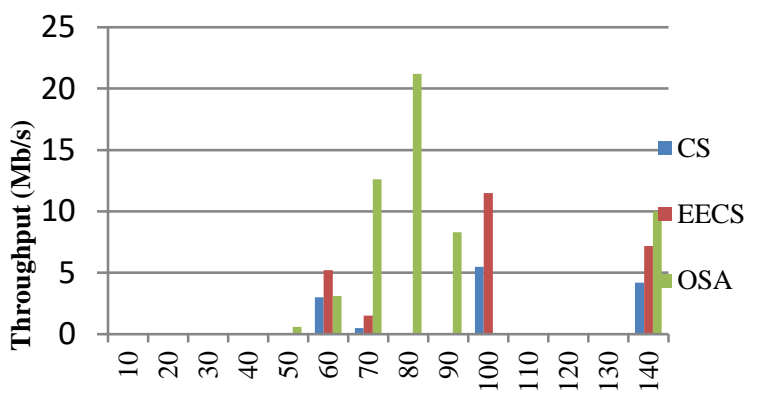

Time (s)

Fig. 15. Throughput of network

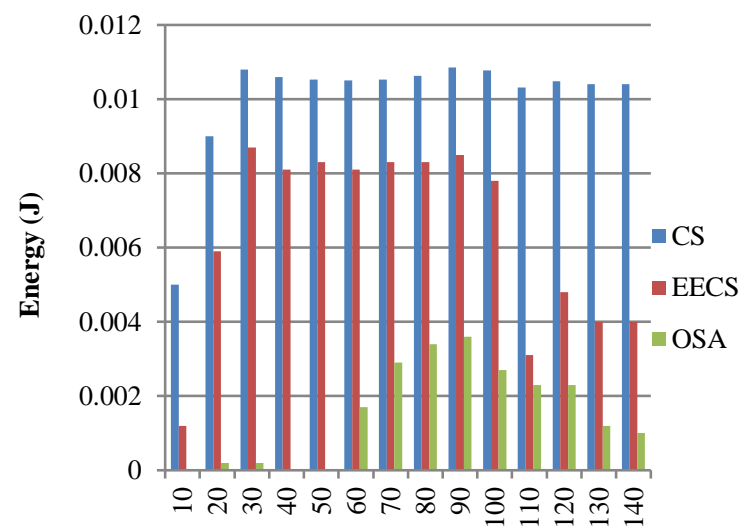

Time (s)

Fig. 16. Energy consumption in network routing

In summary, the experimental results show that our Cognitive radio network strategy is capable of protecting the user data and isolating the primary user's data levels. The cost is small, and there is not significant impact on the performance of the network. In fact, in order to examine the performance in extreme situations, we made all the traffic connections primary user related. Therefore, the performance must be better for normal situations in which there would be connections between normal users. 
Table 2. Simulation parameters

\begin{tabular}{|c|c|}
\hline PARAMETER & VALUE \\
\hline Application Traffic & FTP \\
\hline Transmission rate & 20 packets/sec \\
\hline Radio range & $250 \mathrm{~m}$ \\
\hline Packet size & 512 bytes \\
\hline Maximum speed & $10 \mathrm{~m} / \mathrm{s}$ \\
\hline Simulation time & $150 \mathrm{~s}$ \\
\hline Number of nodes & 10 \\
\hline Area & $1000 x 1000$ \\
\hline Channels/radio & 5 \\
\hline $\begin{array}{c}\text { Maximum number of } \\
\text { packets }\end{array}$ & 10000 \\
\hline Total packets in node & 50 \\
\hline Routing protocol & AODV \\
\hline
\end{tabular}

\section{Conclusion}

In this system, the issue of coordinated relay choice in CRNs is mitigated by optimal selection scheme. We have proposed a solution for auxiliary client $\mathrm{s}$ in CCRNs to maximize SUs' energy efficiency which is contributed by the delay, throughput and energy consumption. Superiority of the scheme is explained by comparing both random selection policy and optimal stopping policy through numerical simulation. The issue of coordinated relay determination is considered here, in which the Primary clients effectively select suitable auxiliary clients as hand-off nodes to improve their transmission execution. In this paper, an optimal selection scheme is proposed to solve the problem of cooperative relay selection in CRNs. Here a primary client pair observes the auxiliary client candidate relays in certain order and selects one as their cooperative relay if the transmission requirement of the auxiliary client is satisfied. An intuitive observation order and proved its optimality from the aspect of efficiency. The proposed method yields low energy consumption and maximum throughput is obtained at minimum scan time. A steady state range is obtained by this relay selection policy. Thus a steady state relay selection policy is obtained. Comparative analysis with existing cooperative approaches presented in the related also provided in the discussion, our proposed method reduces the energy consumption and increases the throughput, which will enhance the system efficiency and robustness.

Moreover, this can be actualized on relay based secondary user (SU) correspondence in subjective radio systems, in which two auxiliary handset nodes trade their data with the help of a transfer under impedance control limit imperatives. The address of node in light of relay selection (RS), power allocation (PA), and physical layer network coding (PNC) chose.

\section{References}

[1] I. F. Akyildiz, W. Y. Lee, M. C. Vuran, and S. Mohanty, "A survey on spectrum management in cognitive radio networks", IEEE Communications Magazine, Vol. 46, No. 4, pp. 40-48, 2008.

[2] K. Davaslioglu and E. Ayanoglu, "Quantifying Potential Energy Efficiency Gain in Green Cellular Wireless Networks", IEEE Communications Surveys \& Tutorials, Vol. 16, No. 4, pp. 2065-2091, 2014.

[3] O. Holland, V. Friderikos, and A. H. Aghvami, "Green spectrum management for mobile operators", In: Proc. of IEEE Globecom Workshops, pp. 1458-1463, 2010.

[4] J. Mitola and G. Q. Maguire, "Cognitive radio: Making software radios more personal", IEEE Pers. Commun., Vol. 6, No. 4, pp. 13-18, 1999.

[5] F. Li, B. Bai, J. Zhang, and K. B. Letaief, "Location-Based Joint Relay Selection and Channel Allocation for Cognitive Radio Networks", In: Proc. of IEEE Global Telecommunications Conference - GLOBECOM 2011, pp. 1-5, 2011.

[6] S. Haykin, "Cognitive radio: Brain-empowered wireless communications", IEEE J. Sel. Areas Commun., Vol. 23, No. 2, pp. 201-220, 2005.

[7] A. Goldsmith, S. A. Jafar, I. Maric, and S. Srinivasa, "Breaking spectrum gridlock with cognitive radios: An information theoretic perspective", In: Proc. IEEE, Vol. 97, No. 5, pp. 894-914, 2009.

[8] O. Simeone, Y. Bar-Ness, and U. Spagnolini, "Stable throughput of cognitive radios with and without relaying capability", IEEE Trans. Commun., Vol. 55, No. 12, pp. 2351-2360, 2007.

[9] Y. Long, H. Li, H. Yue, M. Pan, and Y. Fang, "SUM: spectrum utilization maximization in energy-constrained cooperative cognitive radio networks", IEEE J. Sel. Areas Commun., Vol. 32, No. 11, pp. 2105-2116, 2014.

[10] J. N. Laneman, D. N. C. Tse, and G. W. Wornell, "Cooperative diversity in wireless networks: efficient protocols and outage behavior", IEEE Trans. Inf. Theory, Vol. 50, No. 12, pp. 3062-3080, 2004.

[11] O. Simeone, I. Stanojev, S. Savazzi, Y. BarNess, U. Spagnolini, and R. Pickholtz, "Spectrum leasing to cooperating secondary ad 
hoc networks", IEEE J. Sel. Areas Commun., Vol. 26, No. 1, pp. 203-213, 2008.

[12] J. Zhang and Q. Zhang, "Stackelberg game for utility-based cooperative cognitive radio networks", In: Proc. of the tenth ACM international symposium on Mobile ad hoc networking and computing, pp. 23-32, 2009.

[13] B. Cao, J. W. Mark, Q. Zhang, R. Lu, X. Lin, and X. S. Shen, "On optimal communication strategies for cooperative cognitive radio networking", In: Proc. of IEEE INFOCOM, pp. 1726-1734, 2013.

[14] W. Li, X. Cheng, T. Jing, and X. Xing, "Cooperative multi-hop relaying via network formation games in cognitive radio networks", In: Proc. of IEEE INFOCOM, pp. 971-979, 2013.

[15] T. Jing, S. Zhu, H. Li, X. Cheng, and Y. Huo, "Cooperative Relay Selection in Cognitive Radio Networks", IEEE Transactions on Vehicular Technology, Vol. 64, No. 5, pp. 1872-1881, 2015.

[16] S. K. Jayaweera, M. Bkassiny, and K. A. Avery, "Asymmetric cooperative communications based spectrum leasing via auctions in cognitive radio networks", IEEE Trans. Wireless Commun., Vol. 10, No. 8, pp. 2716-2724, 2011.

[17] J. Zou, Q. Wu, H. Xiong, and C. Chen, "Dynamic spectrum access and power allocation for cooperative cognitive radio networks", IEEE Trans. Signal Process., Vol. 63, No. 21, pp. 5637-5649, 2015.

[18] Q. Zhang, B. Cao, Y. Wang, N. Zhang, X. Lin, and L. Sun, "On exploiting polarization for energy-harvesting enabled cooperative cognitive radio networking", IEEE Wireless Commun., Vol. 20, No. 4, pp. 116-124, 2013.

[19] B. Cao, H. Liang, J. W. Mark, and Q. Zhang, "Exploiting Orthogonally Dual-Polarized Antennas in Cooperative Cognitive Radio Networking", IEEE Journal on Selected Areas in Communications, Vol. 31, No. 11, pp. 23622373, 2013.

[20] J. Ma, G. Zhao, and Y. Li, "Soft combination and detection for cooperative spectrum sensing in cognitive radio networks", IEEE Trans. Wireless Commun., Vol. 7, No. 11, pp. 57615766, 2008.

[21] W. Zhang, R. K. Mallik, and K. B. Letaief, "Optimization of cooperative spectrum sensing with energy detection in cognitive radio networks", IEEE Trans. Wireless Commun., Vol. 8, No. 12, pp. 5761-5766, 2009.

[22] J. Z. Moghaddam, M. Usman, F. Granelli, and H. Farrokhi, "Cognitive Radio and Device-to-
Device Communication: A Cooperative Approach for Disaster Response", IEEE Global Communications Conference, pp. 1-6, 2016.

[23] J. Liu, H. Ding, Y. Cai, H. Yue, Y. Fang, and S. Chen, "An Energy-Efficient Strategy for Secondary Users in Cooperative Cognitive Radio Networks for Green Communications", IEEE Journal on Selected Areas in Communications, Vol. 34, No. 12, pp. 31953207, 2016.

[24] S. Tamilarasan and P. Kumar, "Dynamic resource allocation using priority queue scheduling in multi-hop cognitive radio networks", In: Proc. of IEEE International Conference on Computational Intelligence and Computing Research, pp. 1-5, 2016.

[25] X. Zhang and J. Wang, "Heterogeneous QoSDriven Resource Allocation over MIMOOFDMA Based 5G Cognitive Radio Networks", In: Proc. of IEEE Wireless Communications and Networking Conference, pp. 1-6, 2017. 